\title{
Article \\ Phage Display Preparation of Specific Polypeptides in Atherosclerotic Foam Cells
}

\author{
Xiang Ji ${ }^{1,2,+}$, Dan Liu ${ }^{3,+}$, Feng Wu ${ }^{1,2}$, Yu Cen ${ }^{1}$ and Lan Ma ${ }^{1,2,4,5, * \text { D }}$ \\ 1 Institute of Biopharmaceutical and Health Engineering, Tsinghua Shenzhen International Graduate School, \\ Shenzhen 518055, China; x-ji13@mails.tsinghua.edu.cn (X.J.); wf19@mails.tsinghua.edu.cn (F.W.); \\ cen.yu@sz.tsinghua.edu.cn (Y.C.) \\ 2 School of Life Science, Tsinghua University, Beijing 100084, China \\ 3 School of Health, Zhuhai College of Science and Technology, Zhuhai 519041, China; danieliu091@zcst.edu.cn \\ 4 Tsinghua-Berkeley Shenzhen Institute (TBSI), Shenzhen International Graduate School, Tsinghua University, \\ Shenzhen 518055, China \\ 5 Shenzhen Bay Laboratory, Shenzhen 518055, China \\ * Correspondence: malan@sz.tsinghua.edu.cn; Tel.: +86-755-26033033 \\ + These authors contributed equally to this work.
}

Citation: Ji, X.; Liu, D.; Wu, F.; Cen, Y.; Ma, L. Phage Display Preparation of Specific Polypeptides in Atherosclerotic Foam Cells. Appl. Sci. 2022, 12, 562. https://doi.org/ 10.3390/app12020562

Academic Editor: Hans Peter Deigner

Received: 24 November 2021

Accepted: 1 January 2022

Published: 6 January 2022

Publisher's Note: MDPI stays neutral with regard to jurisdictional claims in published maps and institutional affiliations.

Copyright: (C) 2022 by the authors. Licensee MDPI, Basel, Switzerland. This article is an open access article distributed under the terms and conditions of the Creative Commons Attribution (CC BY) license (https:// creativecommons.org/licenses/by/ $4.0 /)$.

\begin{abstract}
Atherosclerosis and related complications are the most common causes of death in modern societies. Macrophage-derived foam cells play critical roles in the initiation and progression of atherosclerosis. Effective, rapid, and instrument-independent detection in the early stage of chronic atherosclerosis progression could provide an opportunity for early intervention and treatment. Therefore, as a starting point, in this study, we aimed to isolate and prepare foam cell-specific polypeptides using a phage display platform. The six target polypeptides, which were acquired in this study, were evaluated by ELISA and showed strong specificity with foam cells. Streptavidin coupled quantum dots (QDs) were used as fluorescence developing agents, and images of biotin-modified polypeptides specifically binding with foam cells were clearly observed. The polypeptides obtained in this study could lay the foundation for developing a rapid detection kit for early atherosclerosis lesions and could provide new materials for research on the mechanisms of foam cell formation and the development of blocking drugs.
\end{abstract}

Keywords: atherosclerosis; foam cell; phage display; specific polypeptide

\section{Introduction}

Atherosclerotic lesion (atheroma) is a progressive chronic inflammatory disease characterized by a gradual asymmetric focal thickening of the intima of an artery, and hardening of the artery that ultimately leads to a reduction in lumen diameter and potentially results in ischemia following plaque rupture [1]; atherosclerotic complications are the most common causes of death in modern societies. Atherosclerosis is an inflammatory disease with high plasma concentrations of cholesterol, particularly those of low-density lipoprotein (LDL) cholesterol, which is one of the principal risk factors for atherosclerosis [2]. Previous studies have clarified that the process of atherogenesis consists of much more than the accumulation of lipids within the artery wall; atherogenesis involves cells, connective-tissue elements, lipids, and debris [3]. Blood-borne inflammatory and immune cells constitute an essential part of atheroma, with the remainder including vascular endothelial and smooth muscle cells. Atheroma is preceded by a fatty streak, the earliest type of lesion, which is an accumulation of lipid-laden cells beneath the endothelium. Most of these cells in the fatty streak are macrophages, together with some $\mathrm{T}$ cells. Fatty streaks are prevalent in infants and young people, never cause symptoms, and may progress to atheroma or eventually disappear [4]. In the center of an atheroma, foam cells and extracellular lipid droplets form a core region surrounded by a cap of smooth muscle cells and a collagen-rich matrix. 
T cells, macrophages, and mast cells infiltrate the lesion and are particularly abundant in the shoulder region where the atheroma grows [5].

The formation of macrophage foam cells in the intima of arteries is a major hallmark of early-stage atherosclerotic lesions. Uncontrolled uptake of oxidized low-density lipoprotein (ox-LDL), excessive cholesterol esterification, or impaired cholesterol release result in accumulation of cholesterol ester stored as cytoplasmic lipid droplets and subsequently trigger the formation of foam cells [6]. Scavenger receptors (SRs), such as CD36 and SR class A (SR-A), are the principal receptors responsible for the binding and uptake of ox-LDL in macrophages. ATP-binding cassette (ABC) transporter A1(ABCA1), ABCG1, and scavenger receptor BI (SR-BI) mediate cholesterol export from macrophages. Acyl coenzyme A, cholesterol acyltransferase 1 (ACAT1), and neutral cholesteryl ester hydrolase (nCEH) play critical roles in cholesterol esterification [7-9]. The two main contributing factors of the acellular lipid core of advanced atherosclerosis are the accumulation of extracellular lipid and the death of macrophage foam cells. In addition, advanced atherosclerotic lesions develop from smaller lesions, fatty dots, and streaks composed almost entirely of macrophage foam cells [10-13]. From early fatty streak lesions to advanced plaques, macrophage-derived foam cells are integral to the development and progression of atherosclerosis. Thus, early diagnosis of foam cells plays a vital role in atherosclerosis intervention, and therefore, as a starting point, in this study, we aimed to isolate and prepare foam cell-specific polypeptides using a phage display platform, the QD-based fluorescence image also revealed the binding of these prepared polypeptides with foam cells (Figure 1). The polypeptides we obtained in this study could lay the foundation for developing a rapid detection kit for early atherosclerosis lesions and could provide new materials for research on the mechanisms of foam cell formation and the development of blocking drugs.

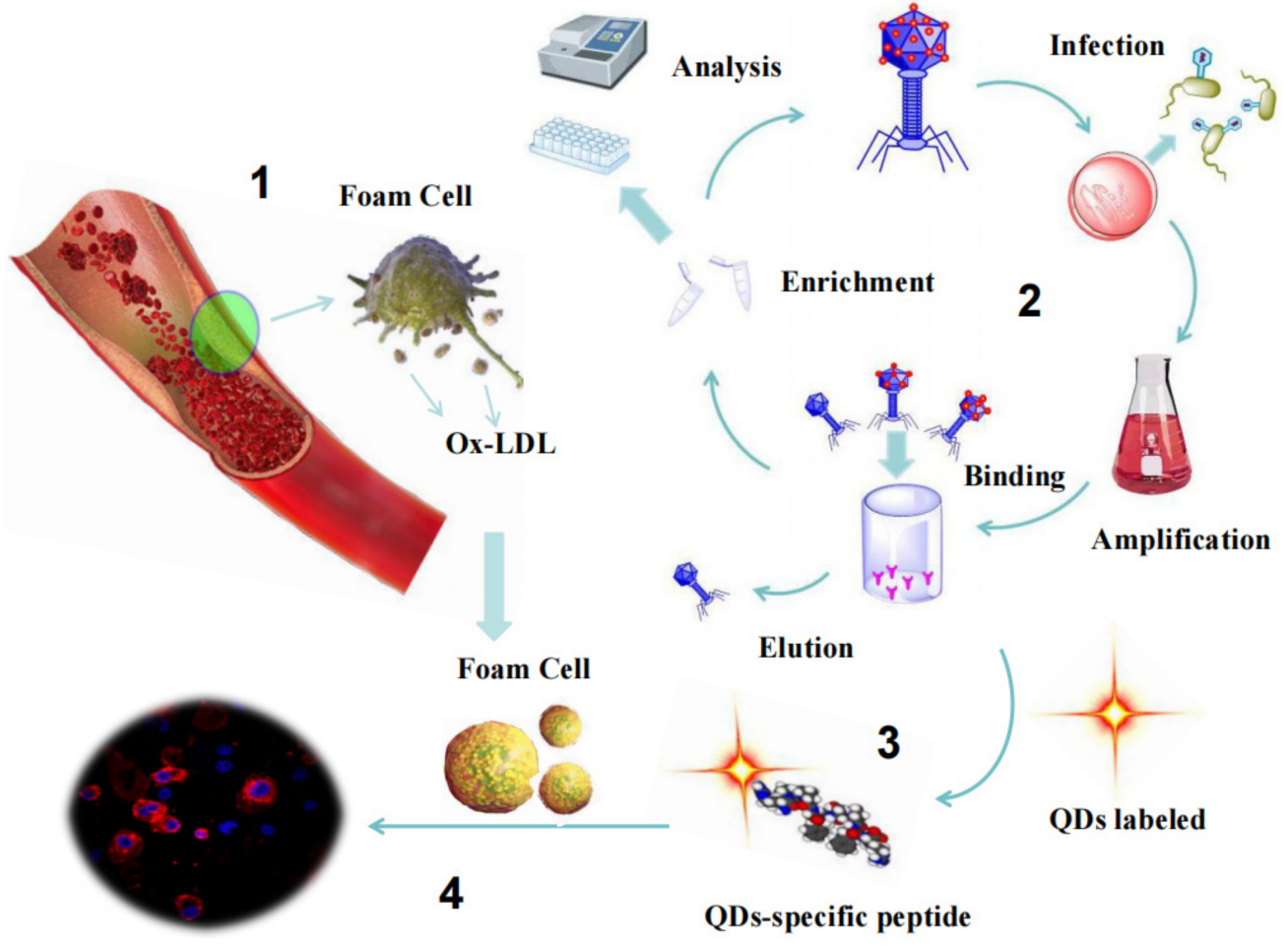

Figure 1. Flow chart of polypeptide preparation by phage display.

\section{Materials and Methods}

\subsection{Materials}

The chemical reagents were all supplied by Sigma-Aldrich; the THP-1 cell lines were purchased from Conservation Genetics ACS Kunming Cell Bank (KCB200549YJ); the Evo M-MLV RT Kit was purchased from Human Accurate Biomedical Technology Co., Ltd. 
Hunan, China; the Reactive Oxygen Species Assay Kit was purchased from Beyotime Biotechnology. Shanghai, China; the SYBR Green PCR Premix HS Taq Kit was purchased from Gen-view Scientific Inc. Hunan, China; and the Ph.D. ${ }^{\mathrm{TM}}$ Phage Display Libraries were supplied by New England BioLabs Inc., Ipswich, MA, USA. The instruments and equipment used included the following: confocal microscope, FV1000 Olympus Fluoview; nanodrop device, Nanodrop 2000 Thermo Scientific; qRT-PCR device, qTower3 Analytik Jena; flow cytometry device, CytoFlex Cytometer Beckman Coulter; microplate reader, Thermo Fisher Scientific Multiskan FC.

\subsection{Methods}

\subsubsection{Establishment of the Foam Cell Model}

The THP- 1 cells were recovered and cultured at a density of $1 \times 10^{6}$ cells $/ \mathrm{mL}$, and the isolated cells were induced by phorbol 12-myristate 13-acetate (PMA) at $160 \mathrm{nM}$ for $24 \mathrm{~h}$. The adherent cells were co-incubated with ox-LDL at $100 \mu \mathrm{g} / \mathrm{L}$ for another $48 \mathrm{~h}$.

\subsubsection{Stain}

\section{Stain of Adherent Cells}

The cell climbing sheets were immersed in $60 \%$ ethanol solution (containing $0.05 \%$ $\mathrm{NaOH}$ ). After slight shaking overnight, the cell slides were washed with deionized water and stored in $75 \%$ ethanol for further use. Cells were plated in 24-well plates at $5 \times 10^{5}$ cells/well; a processed dry cell slide was put in each well and induced by $160 \mathrm{nM}$ PMA at $37^{\circ} \mathrm{C}$ for $24 \mathrm{~h}$, and then co-incubated with ox-LDL at $100 \mu \mathrm{g} / \mathrm{L}$ for another $48 \mathrm{~h}$. After washing with $0.02 \mathrm{M}$ PBS twice, the $10 \%$ neutral methanol solution was added to fix for $30 \mathrm{~min}$, and then washed twice using $0.02 \mathrm{M}$ PBS; $100 \mu \mathrm{L} 0.2 \%$ Oil Red O solution (dissolved in isopropanol/deionized water, 3:2) was added in each wall to stain for $30 \mathrm{~min}$. Next, the cell slides were decolorized using $75 \%$ ethanol for $30 \mathrm{~s}$, and then the cell slides were restained using $0.6 \%$ hematoxylin solution (ethanol/deionized water/glycerin 1:1:1), and washed using PBS. Anti-fluorescence quenching agents were used to block the slides.

\section{Stain of THP-1}

The THP-1 cells were suspended in 0.02 M PBS and centrifuged at $1000 \mathrm{rpm}$, and then the supernatant was removed and $500 \mu \mathrm{L} 10 \%$ neutral methanol solution was added to fix for $30 \mathrm{~min}$ at room temperature. After washing twice with PBS, $500 \mu \mathrm{L} 0.2 \%$ Oil Red O solution was added, for staining for $1 \mathrm{~min}$, and isolated by $1000 \mathrm{rpm}$ centrifugation. Finally, the cells were resuspended in $50 \mu \mathrm{L}$ mounting medium and examined at $\times 200$ magnification by an upright microscope.

\subsection{Detection of ROS in Foam Cells}

The THP-1 was inoculated into a black 96-well microplate and induced to foam cells according to 1. After $200 \mu \mathrm{L}$ of RPMI1640 was added in each well, the DCFH-DA was added, and the final concentration was adjusted to $5 \mu \mathrm{M} /$ well and $10 \mu \mathrm{M} /$ well for both the control group and foam cells, respectively. The cultures were incubated in the cell incubator at $37^{\circ} \mathrm{C}$ for $30 \mathrm{~min}$. The plates were washed three times using PBS, and the fluorescence was detected under 488 and $525 \mathrm{~nm}$ excitation light.

\subsection{Quantitative Real-Time PCR ( $q R T-P C R)$}

Total RNA was extracted with Trizol, purified by centrifugal precipitation, and added as a template to reverse-transcriptase reactions using an Evo M-MLV RT Kit. Quantitative real-time PCRs (qRT-PCRs) were carried out with the resulting cDNAs in triplicate using SYBR Green Pro Taq Hs Premix and a Biorad CFX384 Real-Time System. The experimental $\mathrm{Ct}$ values were normalized to $18 \mathrm{~s}$ and relative mRNA expression was calculated versus a reference $\beta$-actin sample. Each sample was run and analyzed in triplicate. The CD36 primer sequences were CGCTGAGGACAACACAGTCT (forward) and GTTGTCAGCCTCTGTTCCAA (reverse), the SR-B1 primer sequences were 
AACAACTCCGACTCTGGGCTCT (forward) and CATTTGCCCAGAAGTTCCATTG (reverse), the ABCA1 primer sequences were TTCCCGCATTATCTGGAAAGC (forward) and CAAGGTCCATTTCTTGGCTGT (reverse), the ABCG1 primer sequences were CAGGAAGATTAGACACTGTGG (forward) and GAAAGGGGAATGGAGAGAAGA (reverse), the LDLR primer sequences were CCGCAGCGCTGTAGGGGTCTT (forward) and TCATTGCAGACGTGGGAACAGC (reverse), the TNF- $\alpha$ primer sequences were CGAGTGACAAGCCTGTAGC (forward) and GGTGTGGGTGAGGAGCACAT (reverse).

\subsection{Phage Display}

\subsubsection{Screening of Phages}

The Ph.D.-12 phage experienced negative screening and positive screening by THP-1 and foam cells, respectively. In total, 1101 phages were added into $1 \mathrm{~mL} \mathrm{3 \%}$ BSA-PBS suspension containing $1 \times 10^{5}$ THP- 1 cells for incubation, and the rotational speed of the shaker was $150 \mathrm{rpm}$, for $1 \mathrm{~h}$. Then, the mix suspension was centrifuged at $6000 \mathrm{rpm}$ for $5 \mathrm{~min}$; the supernatant was transferred into foam cell culture flasks, which were derived from THP-1, and incubated with same rotation speed for another $1 \mathrm{~h}$. After removing the supernatant, the cells were washed three times with $0.1 \%$ TBST buffer, and then washed another three times using PBS. Then, $1 \mathrm{~mL}$ elution buffer was added, shaking incubation at $200 \mathrm{rpm}$ for $20 \mathrm{~min}$. Then, the mixture was transferred into the centrifuge tube with $150 \mu \mathrm{L}$ neutralization buffer. Finally, the eluted phages were collected by 120,000 rpm high-speed centrifugation. The phage titer was determined by the spot count of phage infected E. coli on an LB solid medium [14].

\subsubsection{Amplification and Purification of the Phage}

In this study, we chose the logarithmic growth of E. coli ER2738 as the host cell and purified by high-speed centrifugation. Specifically, the phage eluate from the screening step was added and incubated at $37^{\circ} \mathrm{C}$ for $4.5 \mathrm{~h}$. The infection bacteria solution was centrifuged $\left(12,000 \mathrm{rpm}, 4^{\circ} \mathrm{C}, 10 \mathrm{~min}\right)$ and the supernatant was transferred into PEG-NaCl (MW 80,000) at a $1 / 6$ volume ration. After $2 \mathrm{~h}$ incubation, the solution was centrifuged $(12,000 \mathrm{rpm}$, $4{ }^{\circ} \mathrm{C}, 20 \mathrm{~min}$ ) again, the precipitate was resuspended using $1 \mathrm{~mL}$ TBS-T, followed by a third centrifugation $\left(12,000 \mathrm{rpm}, 4^{\circ} \mathrm{C}, 5 \mathrm{~min}\right)$. The supernatant was collected in $200 \mu \mathrm{L}$ PEG-NaCl and finally centrifuged $\left(12,000 \mathrm{rpm}, 4^{\circ} \mathrm{C}, 20 \mathrm{~min}\right)$ after $2 \mathrm{~h}$ ice incubation. The precipitate was resuspended using $200 \mu \mathrm{L}$ TBST and stored at $4{ }^{\circ} \mathrm{C}$ for further use.

\subsubsection{Phage-Specific Attachment Assay}

The THP- 1 was induced to foam cell in a 96-well microplate according to Section 2.2.1. After immobilization, using $100 \mu \mathrm{L}$ paraformaldehyde in each well, for $30 \mathrm{~min}$ at RT, the microplate was washed twice using PBS, and $200 \mu \mathrm{L} /$ well blocking buffer was added and incubated for $2 \mathrm{~h}$. Then, the plates were rinsed three times with PBS-T and patted dry; $100 \mu \mathrm{L}$ monoclonal phage amplification supernatant was added in each well and incubated at $37{ }^{\circ} \mathrm{C}$ for $90 \mathrm{~min}$. The plate was washed with $0.3 \%$ Tween-20 PBS-T and PBS, three times in sequence, and $100 \mu \mathrm{L}$ of $0.02 \%$ HRP-M13c antibodies was added and incubated at $37^{\circ} \mathrm{C}$ for $90 \mathrm{~min}$. After another extensive wash with PBS-T, $100 \mathrm{~mL}$ of OPD substrate was added to each well. Plates were incubated at room temperature for $15 \mathrm{~min}$. The absorbance was read at $492 \mathrm{~nm}$ after the reaction was stopped by $2 \mathrm{M} \mathrm{H}_{2} \mathrm{SO}_{4}$ stop solution.

\subsubsection{Flow Cytometry Analysis}

The induced foam cells were washed three times using $0.1 \mathrm{M}$ PBS, and were digested with $1 \mathrm{~mL} 0.05 \%(w / w)$ trypsin-EDTA for $10 \mathrm{~min}$ and stopped by FBS contained medium. After $5 \mathrm{~min} 1000 \mathrm{rpm}$ centrifugation, the supernatant was removed and $1 \mathrm{~mL}$ RPMI1640 suspension cells were added, followed by a DCFH-DA probe $(5 \mu \mathrm{M}$ and $10 \mu \mathrm{M})$ and incubated at $37^{\circ} \mathrm{C}$ for $30 \mathrm{~min}$. The treated cells were collected under $1000 \mathrm{rpm}$ centrifugation for $5 \mathrm{~min}$ and washed three times using 0.1 M PBS. Finally, the suspended cells (filtered 
by 200 mesh) were injected into flow cytometry with $60 \mu \mathrm{L} / \mathrm{min}$ under $525 \mathrm{~nm}$ excitation wavelength. Finally, $10^{4}$ cells were counted as a sample group, and the fluorescence intensity was collected and analyzed.

\section{Results}

\subsection{Foam Cell Model}

In this study, we derived foam cells induced from THP-1 and identified them by cellular physiochemistry, as well as at the molecular level by Oil Red O (ORO) staining, ROS fluorescence analysis, and RT-PCR. Figure 2 shows the representative images of Oil Red O staining for intracellular lipids, in which foam cells appeared to be larger than THP-1, and the dense ORO-stained particles of foam cells showed that they were filled with the most lipid droplets.
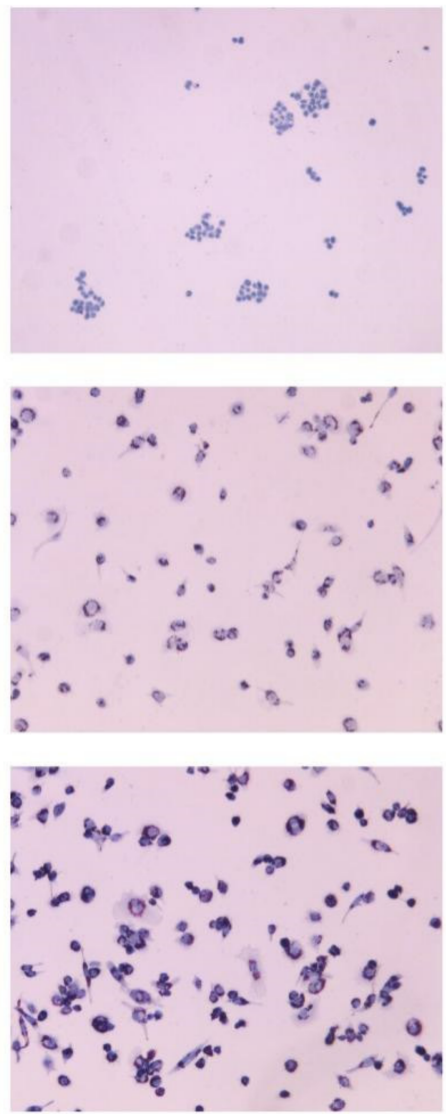

$10 \mathrm{X}$
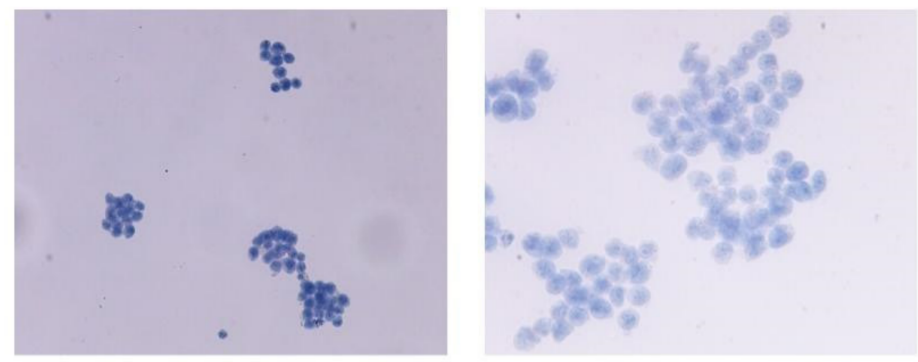

THP-1
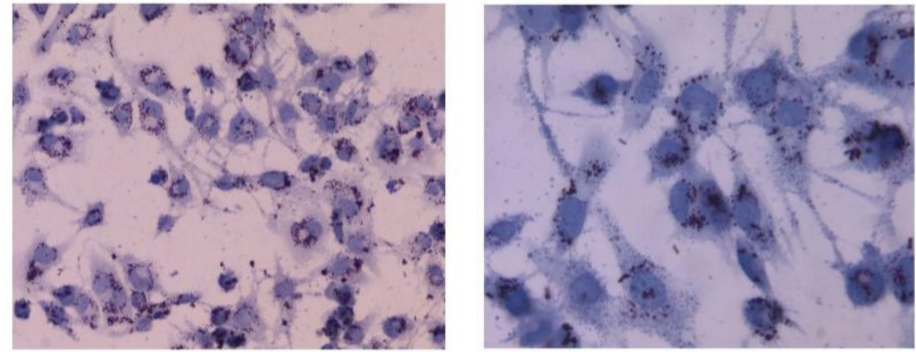

M $\varphi$

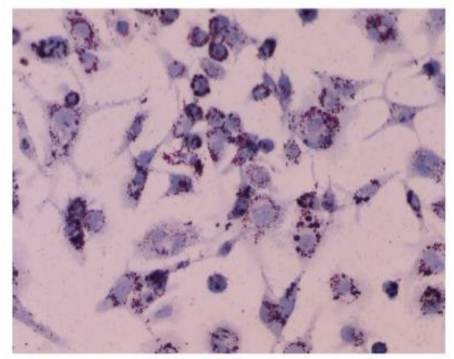

20X

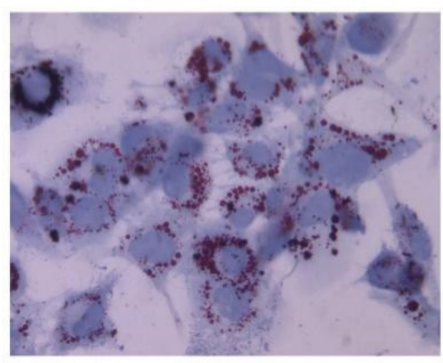

$40 X$

Figure 2. The microphotography of Oil Red O staining: THP-1; macrophage cells $(\mathrm{M} \varphi)$; and foam cells.

The ROS detection was performed using DCF as a fluorescence probe, and the results are shown in Figure 3. The fluorescence signals of foam cells are all higher than THP-1 under $5 \mu \mathrm{M}$ and $10 \mu \mathrm{M}$ DCF treatment (Figure 3a); the flow cytometry shows the same results (Figure 3b); the foam cells' fluorescence intensities (blue curve) are stronger than THP-1 (green curve) on the horizon axis, and 100 times stronger than the control groups. Thus, the ROS detection results show that the foam cells have been successfully induced from THP-1. 

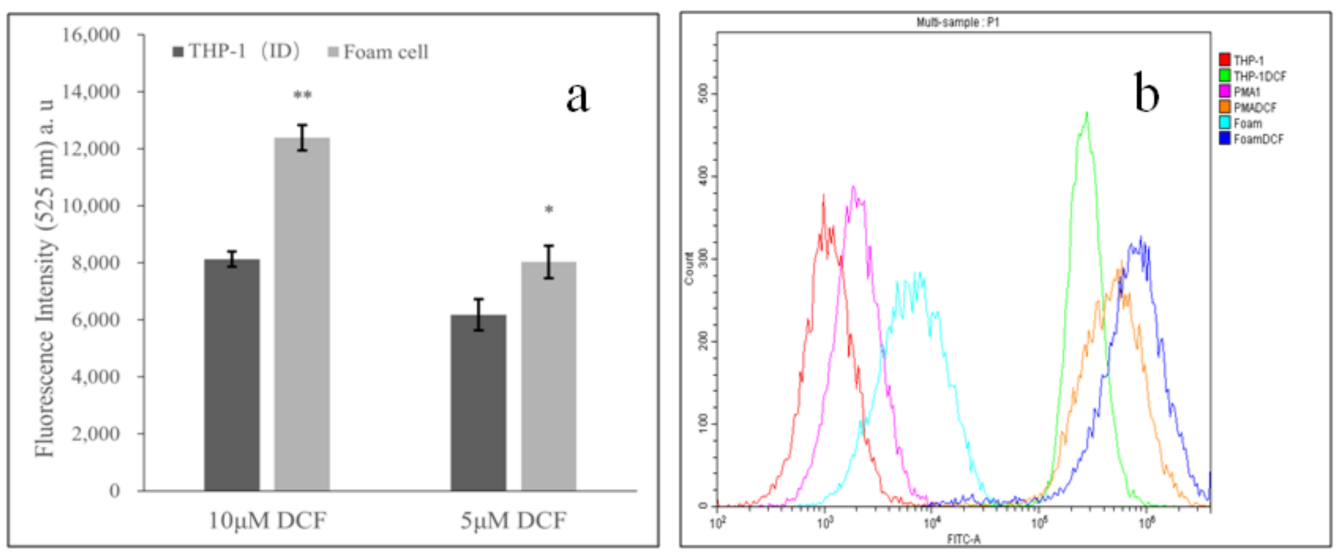

Figure 3. The results of ROS detection in THP-1 and foam cells using DCF fluorescence probe. The fluorescence signals were collected and analyzed by: (a) spectrophotometer (b) flow cytometry. ${ }^{*}$ represents $p$ value range in $t$-test, ${ }^{*}=0.05,{ }^{* *}=0.001$.

The characteristic genetic markers of foam cells, CD36, LDLR, ABCA1, ABCG1, and TNF- $\alpha$ were analyzed by qRT-PCR (Figure 4 ). The results demonstrate that all the detected markers show high expression in foam cells as compared with THP-1. Furthermore, the inflammation-related cytokine TNF- $\alpha$ 's expression is the highest, followed by CD36, and then ABCA1 and LDLR. These results are sufficient to prove that we have successfully established a foam cell model.

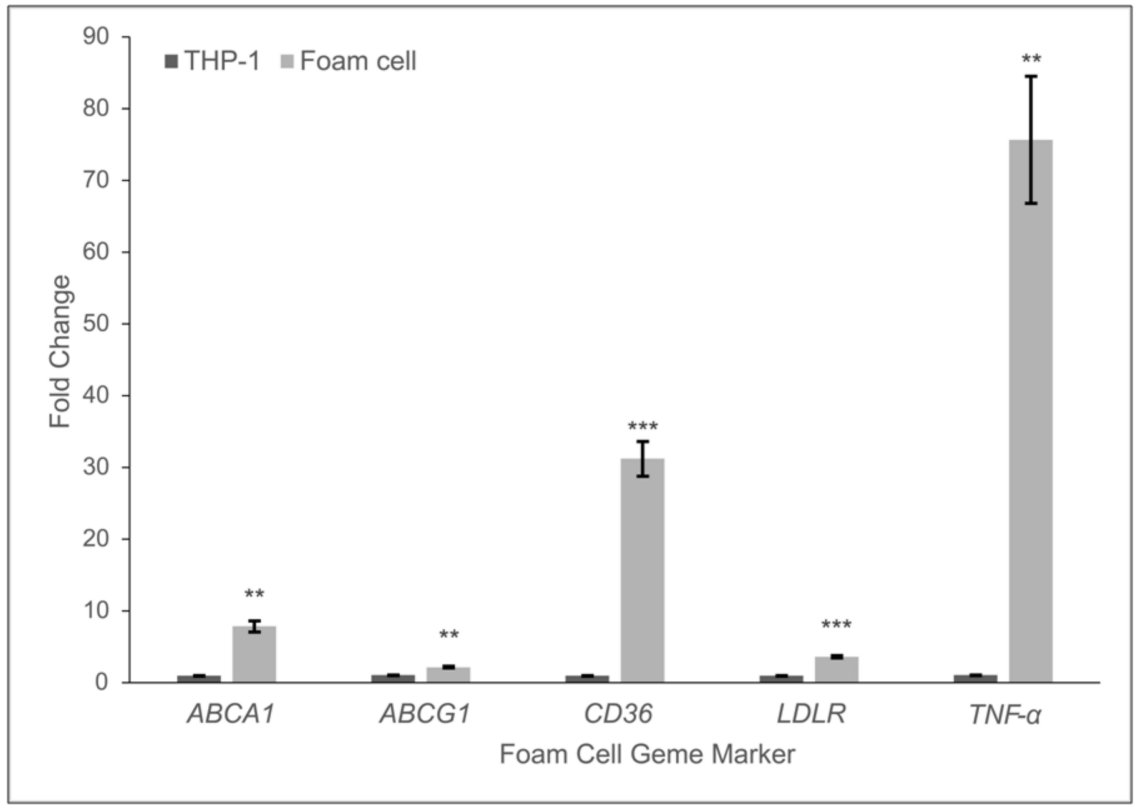

Figure 4. The comparison of genetic markers' expressions between THP-1 and foam cells by qRT-PCR. ${ }^{*}$ represents $p$ value range in $t$-test, ${ }^{* *}=0.001,{ }^{* * *}=0.0001$.

\subsection{Phage Display}

In order to ensure the downstream-desired polypeptides' purity and accuracy, the Ph.D.-12 phages experienced negative screening by THP- 1 and positive screening by foam cells, and only the unbound phages in negative screening were qualified for the next positive screening, and only the eluted phages from foam cells in positive screening could be used for amplifying each cycle (Figure 5). In our study, the amplified phage's titer was measured by gradient dilution-blue plaque counting in each round. The detailed indicators are shown in Table 1. In addition, the enrichment proportion of polypeptide was counted and is displayed in Figure 6. 
Figure 6 illustrates that the specific binding phages were increased significantly as the number of rounds increased, which indirectly confirmed an increase in the specificity of the polypeptides during the foam cell binding process.

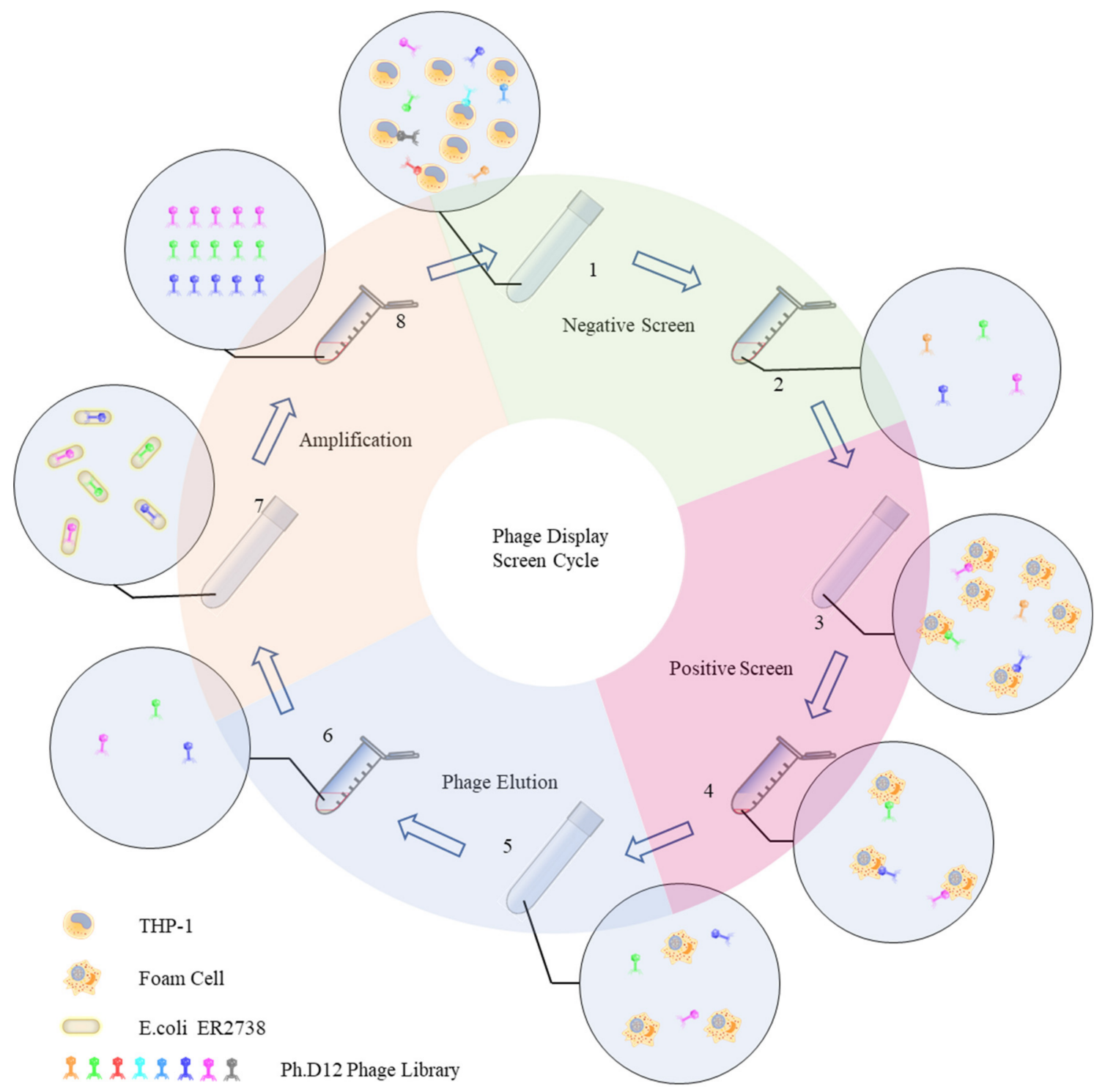

Figure 5. Positive and negative screening in phage display.

Table 1. The amplified phage's titer in each round.

\begin{tabular}{ccccc}
\hline Cycles & Titer & Quantity & Neutralization Titer & Elution Amount \\
\hline 1 & $3.2 \times 10^{9} / \mu \mathrm{L}$ & $10^{11}$ & $1.08 \times 10^{4} / \mu \mathrm{L}$ & $2.16 \times 10^{7}$ \\
2 & $1.0 \times$ & $10^{11}$ & $2.25 \times 10^{5} / \mu \mathrm{L}$ & $4.5 \times 10^{8}$ \\
3 & $10^{10} / \mu \mathrm{L}$ & & $6 \times 10^{5} / \mu \mathrm{L}$ & $1.2 \times 10^{9}$ \\
\hline
\end{tabular}




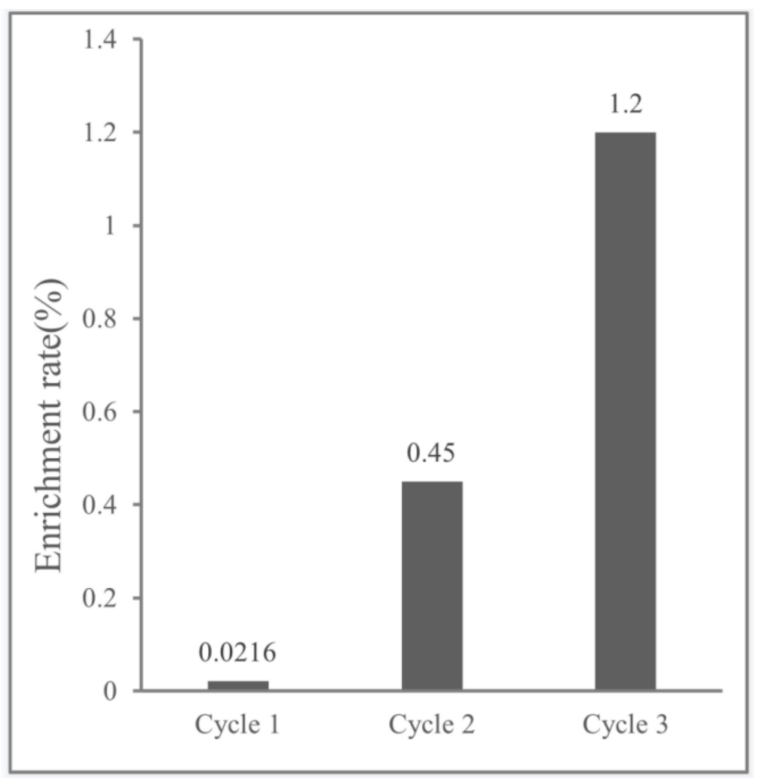

Figure 6. The statistical chart of specific polypeptide's enrichment proportion in each round of phage display.

\subsection{Specific Polypeptides}

Six specific polypeptides were isolated by phage display, and the details are listed in Table 2. Sandwich ELISA was performed to evaluate the specificity of the obtained polypeptides, and the differences between binding to THP- 1 and binding to foam cells were analyzed and compared, as shown in Figure 7. The dark column represents the specificity of binding to foam cells, which are all significantly stronger than the gray column that represents the specificity of THP-1, and proves that the prepared polypeptides have strong specificity with foam cells.

In order to visualize the difference between polypeptide specificity in binding to THP-1 and foam cells, the laser scanning confocal microscope was selected to observe the fluorescence images of polypeptides specifically binding to corresponding cells' processes. The THP-1 and foam cells were both stained using DAPI and incubated with biotinpolypeptide conjugation. The QDs labeled avidin acted as chromogenic agents, and all the treated cells were excited by $365 \mathrm{~nm}$ light. The images are displayed in Figure 8 . The red fluorescence around the blue nucleus stained with DAPI can be seen clearly in all foam cells groups as compared with the THP-1 groups that only showed blue fluorescence with DAPI. These results strongly demonstrate that the isolated polypeptides can specifically and efficiently bind to foam cells.

Table 2. The code name and corresponding sequence of specific polypeptides.

\begin{tabular}{cc}
\hline Code Name & Sequence \\
\hline GAT-T1 & GADTSKPPRFVT \\
SLP-T2 & SLLAERQFNSKP \\
REP-T3 & REDPPTTAYYSP \\
SAH-T4 & SANGETVNPSRH \\
NNN-T5 & NNLPTSRTLAGN \\
VGR-T6 & VGHTVASDIPPR \\
\hline
\end{tabular}




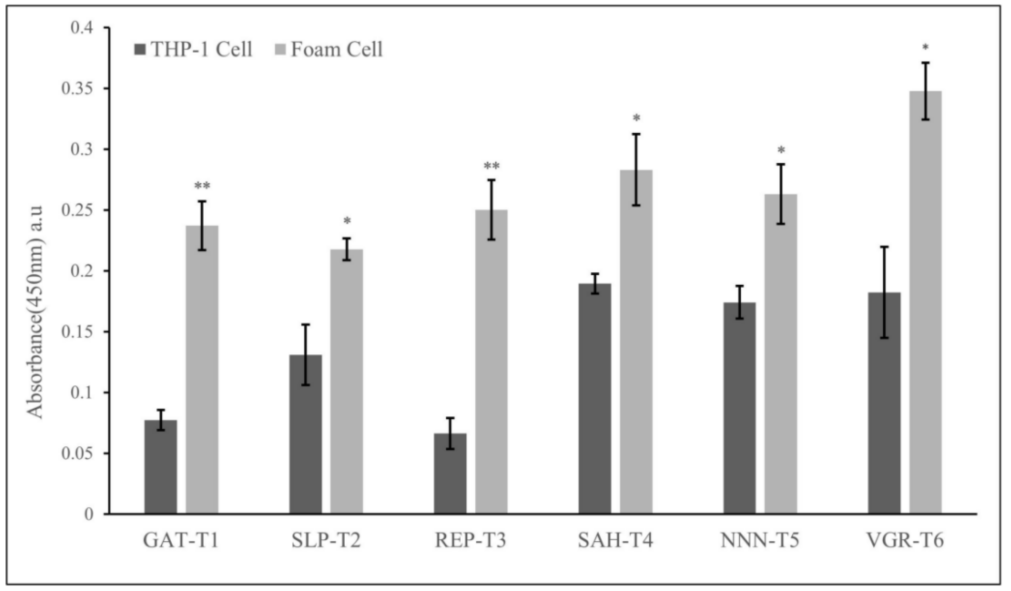

Figure 7. The ELISA results of polypeptide specificity, ${ }^{*}$ represents $p$ value range in $t$-test, ${ }^{*}=0.05$, $* *=0.001$.
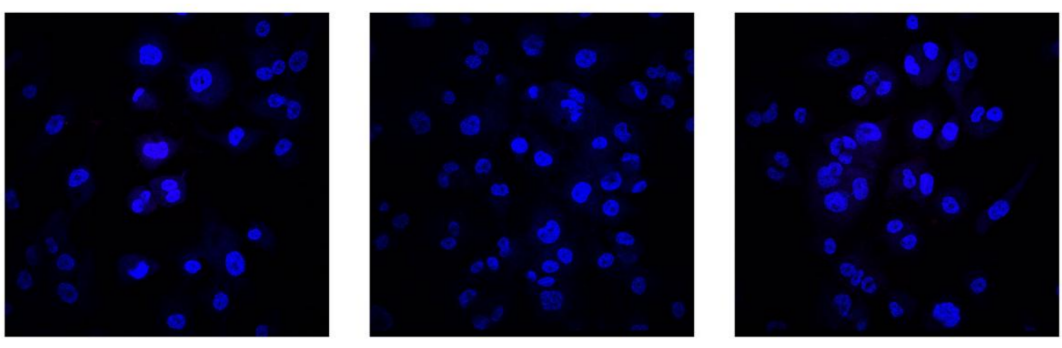

A1
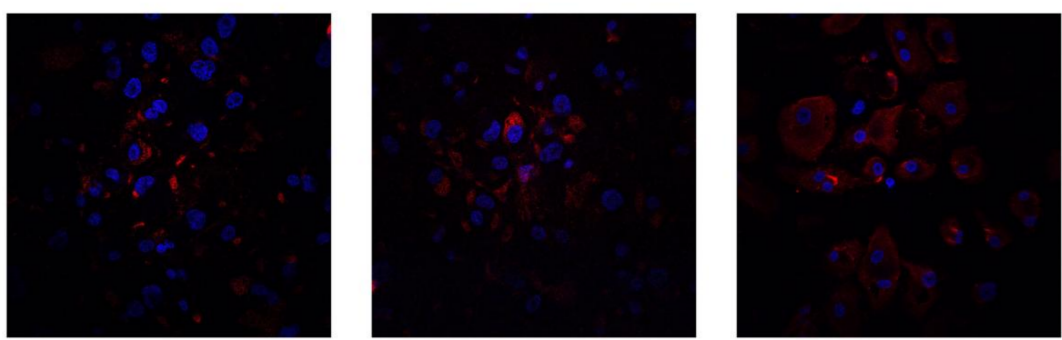

B1

GAT-T1

SLP-T2

REP-T3
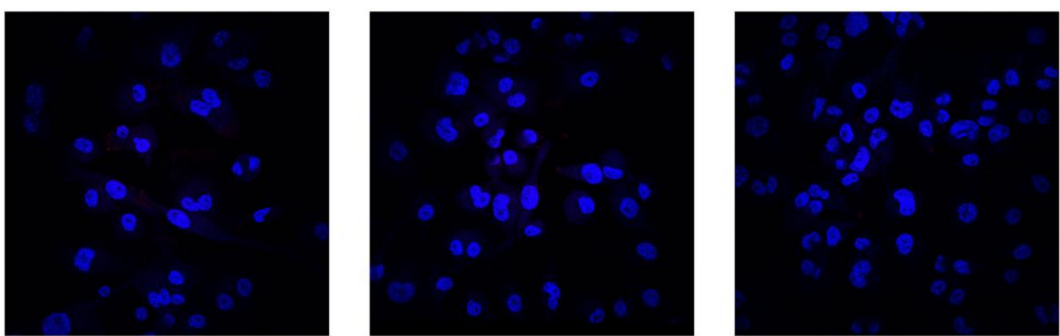

A2

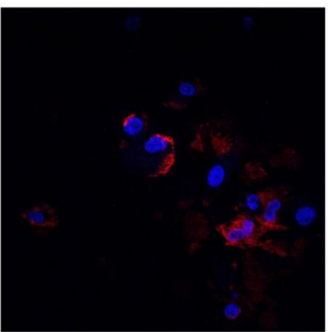

SAH-T4

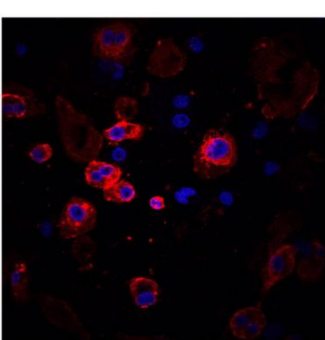

NNN-T5

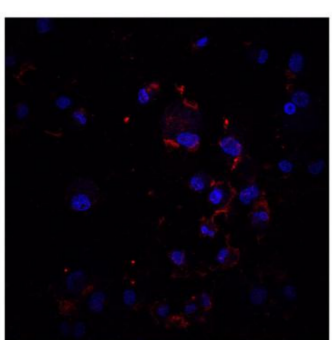

VGR-T6

Figure 8. The confocal images of THP-1 and foam cells, both THP-1 (A1 and A2 rows) and foam cells (B1 and B2 rows) were treated with DAPI and avidin-conjugated QDs. 


\section{Discussion}

Atherosclerosis is a chronic inflammatory disease within vascular walls; the formation of foam cells in a vascular wall is a characteristic feature of atherosclerotic plaques [15]. Monocytes migrate from the circulation into the intima of the arterial wall, where they differentiate into macrophages, which then uptake modified lipoproteins via a relatively large family of scavenger receptors (such as SR-A and CD36), transforming into foam cells [16]. ROS production, such as superoxide, hydrogen peroxide, and peroxynitrite, together with inflammatory factors such as cytokines, chemokines, and adhesion molecules have been shown to be increased in atherosclerotic lesion formation in atherosclerosis [17-19]. Thus, ROS is undoubtedly a representative indicator of foam cell and lesion formation. In this study, we selected a ROS-specific cell-permeable fluorescent probe $2^{\prime}, 7^{\prime}$-dichlorofluorescein diacetate (DCFH-DA) to detect the ROS in foam cells. DCFH-DA is a nonpolar dye which is converted into the polar derivative $2^{\prime}, 7^{\prime}$-dichlorofluorescein (DCFH) by cellular esterase. DCFH is nonfluorescent, very sensitive to several ROS, and can be oxidized to a highly fluorescent $2^{\prime}, 7^{\prime}$-dichlorofluorescein (DCF) when oxidized by intracellular ROS or other peroxides, having an excitation wavelength of $485 \mathrm{~nm}$ and an emission band between 500 and $600 \mathrm{~nm}$ [20]. The fluorescence signal was collected and analyzed by spectrophotometer and flow cytometry in our work (Figure 3). The THP-1 and derived foam cells were both treated with $5 \mu \mathrm{M}$ and $10 \mu \mathrm{M}$ DCFH-DA, respectively. The fluorescence intensity from foam cells is stronger than THP-1 (Figure 3a). In addition, the flow cytometry showed the same results (Figure 3b), i.e., the foam cells' fluorescence intensity (blue curve) is stronger than THP-1 (green curve) on the horizon axis, and 100 times stronger than that of control groups. The ROS detection results show that foam cells have been successfully induced from THP-1.

Oil Red $\mathrm{O}$ is a fat-soluble diazo dye with maximum absorption at $518 \mathrm{~nm}$, which stains neutral lipids and cholesteryl esters but not biological membranes. The hydrophobic Oil Red $\mathrm{O}$ will move from the solvent to associate with the lipid droplets within foam cells [21].

DL receptor (LDLR) is an essential factor for maintaining cholesterol homeostasis. LDLR is a cell-surface receptor that removes the cholesterol-rich LDL from plasma and maintains the cholesterol level in the blood circulation [22]. CD36 functions as a highaffinity receptor for ox-LDL, and binding of ox-LDL to CD36 leads to endocytosis through a lipid raft pathway. Therefore, the pathogenic role of ox-LDL in atherosclerosis largely depends on CD36 [6]. ABCA1 (adenosine triphosphate-binding cassette transporter A1) is a member of the large superfamily of $A B C$ transporters and plays a critical role in the prevention of macrophage foam cell formation and atherosclerosis by mediating the active transport of intracellular cholesterol and phospholipids to apoA-I [23]. Typically, the most widely characterized mechanism by which HDL-C protects against atherosclerosis is reverse cholesterol transport. In this pathway, pivotal steps comprise the ABCA1-mediated cholesterol efflux followed by cholesterol esterification via lecithin-cholesterol acyltransferase $[24,25]$. Thus, ABCA1 expression could be regarded as a macrophage response to cholesterol stress, and overexpression could be correlated with foam cell formation.

Similarly, ABCG1 (adenosine triphosphate-binding cassette transporter G1) mediates cholesterol removal from macrophages to HDL particles but not lipid-free apoA-I. Thus, the formation of foam cells is mainly due to uncontrolled uptake of LDL or impaired cholesterol efflux in macrophages. In this process, CD36 is responsible for the internalization of modified LDL, promoting cellular accumulation of cholesterol. In contrast, the efflux of intracellular cholesterol in macrophages is mediated by reverse cholesterol transporters ABCA1 and ABCG1 [26]. qRT-PCR (Figure 4) showed that the genetic expressions of LDLR, CD36, ABCA1, and ABCG1 in foam cells we induced were all higher than THP-1. It is worth noting that foam cells accumulate lipids and also produce various chemokines and cytokines to induce inflammation in atherosclerotic lesions. Tumor necrosis factor alpha (TNF- $\alpha$ ) is the crucial pro-atherosclerotic cytokines secreted by ox-LDL-activated foam cells. In this experiment, the expression of TNF- $\alpha$ in foam cell was the highest. The above 
results effectively proved, on the basis of molecular biology, that the foam cell model was successfully established.

In 1985, Smith discovered that foreign DNA sequences could be cloned into filamentous bacteriophages such that the cloned sequences were expressed on the surface of the phage particles as fusion proteins. These phage particles could then be enriched for specific sequences based on the binding properties of the displayed fusion proteins $[27,28]$. The peptides in a phage display library have two critical characteristics required for chemical evolution, i.e., replicability and mutability. If the phage's DNA suffers a mutation in the peptide coding sequence, that mutation is passed on to the phage's progeny and can affect the structure of the peptide. Because of its solvent accessibility, a displayed peptide often behaves essentially as it would if it were not attached to the virion surface. Affinity purification has been used to precisely capture phage-displayed peptides from a complex mixture of compounds [29]. Specific binding between polypeptide and foam cells was realized by forming the non-covalent and mediated hydrophobic and Van-der-Waals interactions [30]. This reversible process without bond formation could be disbanded in surfactant solution. In this work, as the pretreatment, the Ph.D.-12 phages experienced negative screening by THP-1, and positive screening by foam cells, to eliminate interference from nonspecific binding and ensure the purity of the target phages; only the unbound phages in negative screening were qualified for the next positive screening, and only the eluted phages from foam cells in positive screening could be used for amplified each cycle. Theoretically speaking, negative screening could ensure that there were no proteins or peptides on the phages' surfaces that could bind to the THP-1, and positive screening could ensure that the phages displayed enough target polypeptides for the following amplification. The principles and process are shown in Figure 5.

Tween-20 was chosen to act as an elution component, and the relationship between elution strength and polypeptide binding affinity was investigated, as shown in Figure 6. In each round of phage display, we gradually increased the concentration of Tween-20 to strengthen the elution force. As a result, the aim of enriched affinity of phages was reinforced by the increasing elution number. However, the specific binding between phage and foam cells was affected by other complicated factors such as eluent resistance and phage proliferation efficiency; these underlying factors were reflected in the decreasing number of plaques after repeatedly screening. Thus, the ELISA analysis was essential to distinguish the target phages. Figure 7 shows the results of target peptides containing phages binding to THP- 1 and foam cells, the six group phages display that the target peptides all obviously strongly bind with foam cells as compared with THP-1, directly proving that the polypeptides we designed and prepared by the phage display platform have high affinity and specificity with foam cells.

In order to further materialize and visualize these experimental results, quantum dots were used to image the processing of polypeptides that specifically bind with foam cells. In our study, a target peptide phage display was designed to label with biotin, and the QDs were conjugated with avidin. When the prepared peptides recognize and bind with foam cells, the exposed biotin residues bind to the streptavidin modified QDs with high affinity, and thus the fluorescence images can be obtained under exciting light by confocal with QDOT625. Figure 8 shows the confocal images of THP- 1 and foam cells, both THP-1 (A row) and foam cells (B row) were simultaneously treated with DAPI and avidin-conjugated QDs. Figure 8 clearly shows that the nuclei are all indistinguishably stained blue by DAPI, and only the foam cells are outlined in red as compared with THP-1. SLP-T2, REP-T3, SAH-T4, and NNN-T5 are particularly obvious in these images. Thus, the confocal results directly demonstrate that specific binding occurred between the polypeptides and the foam cells' surfaces. It is worth mentioning that T5 appears to be much brighter in Figure 8 as compared with T6, but they are inconsistent with the ELISA results in Figure 7. The explanation of this phenomenon is that the aimed peptide fragment of the phage was different from the synthetic peptide fragment as the spatial conformation may change. Specifically, this change, due to the N-terminal and C-terminal, connected the different 
molecules and reflected the different affinities. For this reason, we selected ELISA and immunofluorescence labeled to characterize the affinity of the peptides in parallel, to ensure that the conformation change did not seriously affect the specific affinities between peptides and foam cells. The different affinities between T5 and T6 in Figures 7 and 8 may be derived from the above reason. Moreover, although we tried to use the same parameter settings in the process of collecting fluorescence images, certain errors could have inevitably occurred due to experimental operation and equipment accuracy.

\section{Conclusions}

In this study, a macrophage-derived foam cell model was induced from THP-1 and six specific polypeptides of foam cells were obtained using a phage display platform. These target polypeptides could bind to foam cells with specific and affinity. The QD-based fluorescence image also revealed the binding of these prepared polypeptides with foam cells. This study could lay the foundation for developing a rapid detection kit for early atherosclerosis lesions and could provide new materials for research on the mechanisms of foam cell formation and the development of blocking drugs. The polypeptide that corresponds to ligand protein of foam cells will be studied in future study.

Author Contributions: Conceptualization, L.M. and X.J.; methodology, X.J.; validation, D.L. and F.W.; formal analysis, X.J. and D.L.; investigation, X.J. and L.M.; data curation, D.L., X.J. and F.W.; writing-original draft preparation, D.L.; writing-review and editing, D.L. and X.J.; supervision, L.M. and Y.C.; project administration, L.M. and Y.C.; funding acquisition, L.M. All authors have read and agreed to the published version of the manuscript.

Funding: This work was supported by the National Key R\&D Plan in China (2020YFA0908900), Shenzhen Science and Technology research and development funds (WDZC20200821104802001 and JCYJ20200109143018683), and the State Key Laboratory of Chemical Oncogenomics, Institute of Biomedical Health Technology and Engineering, Shenzhen Bay Laboratory.

Institutional Review Board Statement: This study did not involve animal experiments.

Informed Consent Statement: Not applicable.

Data Availability Statement: The data presented in this study are available on request from the corresponding author.

Acknowledgments: We thank the Tsinghua Shenzhen International Graduate School and Tsinghua Testing Technology Center of Materials and Devices for its generous support of necessary experimental instruments.

Conflicts of Interest: The authors declare no competing financial or commercial interests.

\section{References}

1. Stary, H.C.; Chandler, A.B.; Dinsmore, R.E.; Fuster, V.; Glagov, S.; Insull, W., Jr.; Rosenfeld, M.E.; Schwartz, C.J.; Wagner, W.D.; Wissler, R.W. A definition of advanced types of atherosclerotic lesions and a histological classification of atherosclerosis. Circulation 1995, 92, 1355-1374. [CrossRef]

2. Liuzzo, G. Atherosclerosis-An inflammatory disease. N. Engl. J. Med. 2001, 4, 221-230. [CrossRef]

3. Shepherd, J.; Cobbe, S.M.; Ford, I.; Isles, C.G.; Lorimer, A.R.; MacFarlane, P.W.; McKillop, J.H.; Packard, C.J. Prevention of Coronary Heart Disease With Pravastatin in Men With Hypercholesterolemia. N. Eng. J. Med. 1995, 333, 1301-1307. [CrossRef]

4. Stary, H.C.; Chandler, A.B.; Glagov, S.; Guyton, J.R.; Insull, W., Jr.; Rosenfeld, M.E.; Schaffer, S.A.; Schwartz, C.J.; Wagner, W.D.; Wissler, R.W. A definition of initial, fatty streak, and intermediate lesions of atherosclerosis. A report from the Committee on Vascular Lesions of the Council on Arteriosclerosis, American Heart Association. Circulation 1994, 89, 2462-2478. [CrossRef]

5. de Tena, J.G. Inflammation, atherosclerosis, and coronary artery disease. N. Engl. J. Med. 2005, 353, 429-430. [CrossRef]

6. Yu, X.-H.; Fu, Y.-C.; Zhang, D.-W.; Yin, K.; Tang, C.-K. Foam cells in atherosclerosis. Clin. Chim. Acta 2013, 424, 245-252. [CrossRef] [PubMed]

7. Collot-Teixeira, S.; Martin, J.F.; McDermott-Roe, C.; McDermott-Roe, C.F.; Poston, R.; Poston, R.F.; McGregor, J.L.; McGregor, J.L. CD36 and macrophages in atherosclerosis. Cardiovasc. Res. 2007, 75, 468-477. [CrossRef] [PubMed]

8. Ghosh, S.; Zhao, B.; Bie, J.; Song, J. Macrophage cholesteryl ester mobilization and atherosclerosis. Vascul. Pharmacol. 2010, 52, 1-10. [CrossRef] [PubMed] 
9. Jessup, W.; Gelissen, I.C.; Gaus, K.; Kritharides, L. Roles of ATP binding cassette transporters A1 and G1, scavenger receptor BI and membrane lipid domains in cholesterol export from macrophages. Curr. Opin. Lipidol. 2006, 17, 247-257. [CrossRef]

10. Ball, R.Y.; Stowers, E.C.; Burton, J.H.; Cary NR, B.; Skepper, J.N.; Mitchinson, M.J. Evidence that the death of macrophage foam cells contributes to the lipid core of atheroma. Atherosclerosis 1995, 114, 45-54. [CrossRef]

11. Guyton, J.; Klemp, K. Transitional features in human atherosclerosis. Intimal thickening, cholesterol clefts, and cell loss in human aortic fatty streaks. Am. J. Pathol. 1993, 143, 1444-1457.

12. Hegyi, L.; Skepper, J.F.; Cary, N.R.; Cary, N.F.; Mitchinson, M.J.; Mitchinson, M.J. Foam cell apoptosis and the development of the lipid core of human atherosclerosis. J. Pathol. 1996, 180, 423-429. [CrossRef]

13. Wu, M.; Liu, M.; Guo, G.; Zhang, W.; Liu, L. Polydatin Inhibits Formation of Macrophage-Derived Foam Cells. Evid. Based Complement Alternat. Med. 2015, 729017. [CrossRef]

14. Koch, A.L. The dynamics of coliphage plaque formation. I. Macroplaque experiments. Virology 1959, 8, 273-292. [CrossRef]

15. Hansson, G.K.; Libby, P. The immune response in atherosclerosis: A double-edged sword. Nat. Rev. Immunol. 2006, 6, 508-519. [CrossRef] [PubMed]

16. Liu, W.; Yin, Y.; Zhou, Z.; He, M.; Dai, Y. OxLDL-induced IL-1 beta secretion promoting foam cells formation was mainly via CD36 mediated ROS production leading to NLRP3 inflammasome activation. Inflamm. Res. 2014, 63, 33-43. [CrossRef]

17. Fortuño, A.; San José, G.; Moreno, M.U.; Díez, J.; Zalba, G. Oxidative stress and vascular remodelling. Exp. Physiol. 2005, 90, 457-462. [CrossRef]

18. Gu, L.; Bai, W.; Li, S.; Zhang, Y.; Han, Y.; Yue, G.; Meng, G.; Xie, L.; Jing, W.; Xiao, Y. Celastrol prevents atherosclerosis via inhibiting LOX-1 and oxidative stress. PLoS ONE 2013, 8, e65477. [CrossRef]

19. Deguchi, J.; Aikawa, M.; Tung, C.; Aikawa, E.; Kim, D.; Ntziachristos, V.; Weissleder, R.; Libby, P. Inflammation in Atherosclerosis: Visualizing matrix metalloproteinase action in macrophages in vivo. Circulation 2006, 114, 55-62. [CrossRef]

20. Rastogi, R.P.; Singh, S.P.; Häder, D.-P.; Sinha, R.P. Detection of reactive oxygen species (ROS) by the oxidant-sensing probe $2^{\prime}, 7^{\prime}$-dichlorodihydrofluorescein diacetate in the cyanobacterium Anabaena variabilis PCC 7937. Biochem. Biophys. Res. Commun. 2010, 397, 603-607. [CrossRef]

21. Marquez, V.; Beccaria, A. Application of Oil Red O staining in oleaginous microalgae: Practical uses in screening and lipid quantification of different species. J. Appl. Phycol. 2020, 32, 1755-1761. [CrossRef]

22. Zhong, S.; Li, L.; Zhang, Y.; Zhang, L.; Lu, J.; Guo, S.; Liang, N.; Ge, J.; Zhu, M.; Tao, Y.; et al. Acetaldehyde dehydrogenase 2 interactions with LDLR and AMPK regulate foam cell formation. J. Clin. Investig. 2019, 129, 252-267. [CrossRef]

23. Bochem, A.E.; van Wijk, D.F.; Holleboom, A.G.; Duivenvoorden, R.; Motazacker, M.M.; Dallinga-Thie, G.M.; de Groot, E.; Kastelein, J.J.; Nederveen, A.J.; Hovingh, G.K.; et al. ABCA1 mutation carriers with low high-density lipoprotein cholesterol are characterized by a larger atherosclerotic burden. Eur. Heart J. 2013, 34, 286-291. [CrossRef] [PubMed]

24. Wang, X.; Collins, H.L.; Ranalletta, M.; Fuki, I.V.; Billheimer, J.T.; Rothblat, G.H.; Tall, A.R.; Rader, D.J. Macrophage ABCA1 and ABCG1, but not SR-BI, promote macrophage reverse cholesterol transport in vivo. J. Clin. Investig. 2007, 117, 2216-2224. [CrossRef]

25. Wang, N.; Silver, D.L.; Costet, P.; Tall, A.R. Specific binding of ApoA-I, enhanced cholesterol efflux, and altered plasma membrane morphology in cells expressing ABC1. J. Biol. Chem. 2000, 275, 33053-33058. [CrossRef]

26. Zeng, Y.; Peng, Y.; Tang, K.; Wang, Y.Q.; Zhao, Z.Y.; Wei, X.Y.; Xu, X.L. Dihydromyricetin ameliorates foam cell formation via LXR $\alpha$-ABCA1/ABCG1-dependent cholesterol efflux in macrophages. Biomed Pharmacother. 2018, 101, 543-552. [CrossRef]

27. Smith, G.P. Filamentous fusion phage: Novel expression vectors that display cloned antigens on the virion surface. Science 1985, 228, 1315-1317. [CrossRef]

28. Lonberg, N. Fully human antibodies from transgenic mouse and phage display platforms. Curr. Opin. Immunol. 2008, 20, 450-459. [CrossRef] [PubMed]

29. Smith, G.P.; Petrenko, V.A. Phage Display. Chem. Rev. 1997, 97, 391-410. [CrossRef] [PubMed]

30. Houk, K.N.; Leach, A.G.; Kim, S.P.; Zhang, X. Binding Affinities of Host-Guest, Protein-Ligand, and Protein-Transition-State Complexes. Angew. Chem. 2003, 42, 4872-4897. [CrossRef] [PubMed] 